\title{
VALIDATION OF THE SPECTROFOTOMETRIC METHOD FOR THE DETERMINATION OF QUANTITATIVE COMPOSITION OF S-2,6-DIAGENOXIC ACID OF 3-METHYL-1,2,4-TRIAZOLYL- 5-THIOACETATE
}

\author{
KUCHERENKO LYUDMILA IVANOVNA ${ }^{1,2}$, BIDNENKO OLEKSANDR SERGEEVICH ${ }^{1}$, \\ KHROMYLOVA OLGA VLADIMIROVNA ${ }^{1}$
}

\begin{abstract}
${ }^{1}$ Department of Pharmaceutical Chemistry, Zaporizhia State Medical University, Zaporizhia, Ukraine, ${ }^{2}$ Scientific and Production Association “Farmatron," Zaporizhia, Ukraine. Email: bidnenko2012@gmail.com
\end{abstract}

Received: 13 April 2018, Revised and Accepted: 11 May 2018

\begin{abstract}
Objective: The aim of the work was to determine the validation characteristics for the designed procedure for quantitation of the (S)-2,6diaminohexanoic acid substance, 3-methyl-1,2,4-triazolyl-5-thioacetate, by absorption spectrophotometry in the ultraviolet region.

Methods: For study purposes, the substance (S)-2,6-diaminohexanoic acid of 3-methyl-1,2,4-triazolyl-5-thioacetate and a standard sample (SS) obtained from the State Enterprise "Chemical Reagents Plant" of the Scientific-Technological Complex "Institute of Single Crystals" of the National Academy of Sciences of Ukraine, was used. In the course of our work, we used chemical, physical, physicochemical (absorption spectrophotometry in the ultraviolet region), and statistical methods of analysis. The study was carried out at the Laboratory for Standardization and Technology of Medicines at the Department of Pharmaceutical Chemistry at the Zaporizhia State Medical University (Ukraine).

Results: In previous studies, we have developed a technique for spectrophotometric determination of the quantitative composition of (S)-2,6diaminohexanoic acid of 3-methyl-1,2,4-triazolyl-5-thioacetate. The results of the conducted studies showed that the absorption curve in the UV region of the substance solution has a maximum absorption, namely: $\lambda=238 \mathrm{~nm}$. The results of the quantitation of the substance (S)-2,6-diaminohexanoic acid of 3-methyl-1,2,4-triazolyl-5-thioacetate prove that our technique is accurate and reproducible and meets the current requirements. In order for the developed methodology to ensure consistent and accurate analysis results, its validation was carried out. According to the requirements of the State Pharmacopoeia of Ukraine (DFU) (2 editions), the developed method for the quantitation of the substance was checked for such validation characteristics as specificity, linearity, range of use, accuracy, correctness, and robustness.
\end{abstract}

Conclusion: As a result of the work, it was proved that the developed method of the quantitation of the substance (S)-2,6-diaminohexanoic acid of 3-methyl-1,2,4-triazolyl-5-thioacetate by spectrophotometric method is valid.

Keywords: Ischemic heart disease, Spectrophotometry, Quantitation, Validation, (S)-2,6-diaminohexanoic acid 3-methyl-1,2,4-triazolyl-5-thioacetate. (C) 2018 The Authors. Published by Innovare Academic Sciences Pvt Ltd. This is an open access article under the CC BY license (http://creativecommons. org/licenses/by/4. 0/) DOI: http://dx.doi.org/10.22159/ajpcr.2018.v11i9.26684

\section{INTRODUCTION}

Cardiovascular diseases are fairly called the epidemic of the $20^{\text {th }}$ century. For years, they are the leading cause of mortality in many developed countries, including Ukraine accounting for $55 \%$ of total mortality.

In the pathogenesis of acute focal cerebral ischemia, the primary importance belongs to the reduction of cerebral blood flow and development of circulatory hypoxia due to insufficient income of oxygen and glucose in the nervous tissue. The most promising methods of preventing irreversible damage to the brain substance in patients with ischemic stroke are to restore local cerebral blood flow (thrombolytic therapy) and metabolic brain protection and neuroprotection.

An important element in solving this complex problem is to create new highly effective and safe drugs, the use of which would lead to lower mortality and improve the quality of life.

Numerous experimental data and results of clinical usage of thiotriazoline and its combined dosage forms allowed revealing structural fragments of molecules that determine the presence and strength of anti-ischemic, antioxidant, neuroprotective, and cardioprotective activity. Analysis of the relationship "structureactivity" allowed to determine the perspective direction of creating new biologically active compounds based on 1,2,4-triazole [1].
The staff of the Department of Pharmaceutical Chemistry of the Zaporizhia State Medical University, in cooperation with the specialists of the Scientific-Production Association "Pharmatron," under the leadership of Professor Mazur I.A. during the chemical modification of the drug molecule - the leader of the thiotriazoline, new cationic anionic active compounds were obtained. The most active ingredient is a compound that combines the structural fragments of thiotriazolin and the amino acid of lysine in its molecule, called "Angiolin" - (S)-2,6diaminohexanoic acid 3-methyl-1,2,4-triazolyl-5-thioacetate. (S)-2,6diaminohexanoic acid 3-methyl-1,2,4-triazolyl-5-thioacetate shows cardioprotective, anti-ischemic, antioxidant, and neuroprotective properties with pronounced effects on the endothelium of the vessels of the brain and heart.

In our previous studies, a technique for spectrophotometric determination of the quantitative composition of (S)-2,6diaminohexanoic acid of 3-methyl-1,2,4-triazolyl-5-thioacetate was developed. Therefore, the aim of our work was to determine the validation characteristics for the developed method of quantitation of the substance (S)-2,6-diaminohexanoic acid of 3-methyl-1,2,4-triazolyl5 -thioacetate by absorption spectrophotometry in the ultraviolet region.

\section{METHODS}

For study purposes, the substance (S)-2,6-diaminohexanoic acid of 3-methyl-1,2,4-triazolyl-5-thioacetate and a standard sample (SZ) 
obtained from the State Enterprise "Chemical Reagents Plant" of the Scientific-Technological Complex "Institute of Single Crystals" of the National Academy of Sciences of Ukraine was used. In the course of our work, we used chemical, physical, and physical-chemical (absorption spectrophotometry in the ultraviolet region and absorption spectrophotometry in the infrared region) analysis methods. The study was carried out at the Laboratory for Standardization and Technology of Medicines at the Department of Pharmaceutical Chemistry of the ZSMU.

\section{RESULTS AND DISCUSSION}

In previous studies, we have developed a technique for spectrophotometric determination of the quantitative composition of (S)-2,6-diaminohexanoic acid of 3-methyl-1,2,4-triazolyl-5-thioacetate. The results of the studies showed that the absorption curve in the UV region of the substance solution has the absorption maxima, namely: $\lambda=238 \mathrm{~nm}$. The results of the quantitative determination of the substance (S)-2,6-diaminohexanoic acid of 3-methyl-1,2,4-triazolyl5 -thioacetate spectrophotometrically prove that our technique is accurate and reproducible and meets the current requirements [2,3].

For a high degree of assurance that the substance meets the preestablished eligibility criteria; in particular, validation of technological processes is carried out using samples of at least three series of real product to confirm and provide a documentary evidence that the process (within the established parameters) has a repeatability and leads to the expected results in the production of semi-finished product or finished product of the required quality; validation of analytical methods is to determine accuracy, reproducibility, sensitivity, stability, linearity, and other metrological characteristics.

Testing the stability of the reference solution is one of the elements of studying the robustness of the technique and should be carried out before the start of all other validation studies. As a result of our study, it has been proved that our solution is stable for at least an hour. For this, a parallel measurement of the optical density of the solution was performed at different times $t=0,15,30,45$, and $60 \mathrm{~min}$. The results of testing the stability of our solution at different times are shown in Table 1.

The linear dependence was studied within the range of use of the developed techniques. It was confirmed directly on tablets by dilution of standard solutions (nine concentrations were used). Based on the obtained data, the graphs of the dependence of the optical density of the solutions obtained from their concentration were made. The obtained results were processed using the least squares method, and the linear regression equations of general form were calculated: $y=a+b \cdot x$, where $\mathrm{y}$ - is the measured value (optical density), $\mathrm{x}$ - is the concentration of the determined medicinal substance, a - is a free line of linear dependence for the calculated regression line, and $\mathrm{b}$ - is the angular coefficient for the calculated regression line. To quantify the reproducibility of the results of the definitions and the adequacy of the linear dependence, the numerical values of the linear dependence were calculated, namely, the correlation coefficient $\mathrm{R}$ (should be greater than the general correlation index Rc), the residual standard deviation S0 (should not exceed the maximum uncertainty of the analysis $\Delta \mathrm{As}$ ), the free member of the linear regression a $(|a| \leq \Delta a)$ and regression $b$ coefficient, and linear dependence are shown in Table 2.

The calculated numerical values of linear dependence indicate that the linearity meets the requirements of the DFU in the selected concentration ranges (Fig. 1).

According to the requirements of the DFU, such validation characteristics as the correctness and accuracy of the developed methods for quantitation of the active substance in the drugs composition were established by the method of model mixtures (Table 3). The general objective in determining the correctness of the techniques is to identify possible systematic errors that arise as a result of influencing the results of definitions of excipients that are part of the drug $[4,5]$

Precision (intra-laboratory precision) of the technique is determined by the proximity of the results for a series of measurements carried out according to this method in different samples of the same homogeneous sample and is conditioned by the presence of random errors. According to the DFU, the precision of the technique is considered at three levels as follows: Intralaboratory precision, convergence, and reproducibility [6-8]. In this study, the precision of the proposed method was determined on the level of convergence. To do this, in each case, 15 parallel determinations were carried out (five weighs and three repetitions), and metrological characteristics were determined according to the results. According to the data shown in Table 4, it is established that in all cases the one-way confidence interval does not exceed the maximum permissible uncertainty of the analysis; therefore, the methods are accurate at the level of convergence.

The range of use of the analytical technique is the interval between the minimum and maximum concentrations of the test substance, for which it is shown that the technique has the required linearity, correctness, and precision. According to the results of the carried out study, usable ranges for the developed techniques are within the working intervals for the methods of quantitation according to the DFU requirements $(80-120 \%)[9,10]$.

The assessment of workability was carried out at the stage of development of techniques by identifying factors that influence the

Table 1: Stability of the solution of (S)-2,6-diaminohexanoic acid of 3-methyl-1,2,4-triazolyl-5-thioacetate

\begin{tabular}{|c|c|c|c|c|c|c|c|c|c|}
\hline$t, \min$ & 0 & 15 & 30 & 45 & 60 & Mean & RSDt \% & $\Delta \mathrm{t} \%$ & $\max$ \\
\hline $\mathrm{A} 0$ & 0.485 & 0.485 & 0.486 & 0.486 & 0.486 & 0.486 & 0.11 & 0.23 & 0.56 \\
\hline A & 0.481 & 0.481 & 0.482 & 0.482 & 0.483 & 0.482 & 0.17 & 0.30 & 0.56 \\
\hline
\end{tabular}

RSD: Relative Standard deviation

Table 2: Metrological characteristics of linear dependence $y=a+b x$

\begin{tabular}{llll}
\hline Value name & Data value & Criteria (for 98.5-101.0\% allowance, number of points 9) & Result (match or does not match) \\
\hline $\mathrm{b}$ & 1.0206 & - & - \\
$\mathrm{Sb}$ & 0.0109 & - & - \\
$\mathrm{a}$ & 1.9315 & $\leq 1.8946^{*} \mathrm{~S}_{\mathrm{a}}=0.14 ;$ & correspond \\
& & If it does not realize 1), & - \\
$\mathrm{Sa}$ & 0.0776 & then $\leq 2.5$ & - \\
$\mathrm{Sr}$ & 0.9992 & $\leq 1.24$ & correspond \\
$\mathrm{R}$ & 0.9996 & $\geq 0.99535$ & \\
\hline
\end{tabular}


Table 3: Results of analysis of model solutions and their statistical processing

\begin{tabular}{|c|c|c|c|c|c|}
\hline $\begin{array}{l}\text { No of model } \\
\text { solutions }\end{array}$ & $\begin{array}{l}\text { Weighed portion } \\
\text { of substation, } \\
g\left(m_{s t}=0,0500\right)\end{array}$ & $\begin{array}{l}\text { Presented in } \% \text { to substance } \\
\text { concentration of the } \\
\text { comparison - } \mathrm{X}_{\mathrm{i}} \text {, fact, } \%\end{array}$ & $\begin{array}{l}\text { Average optical } \\
\text { density }(0.386)\end{array}$ & $\begin{array}{l}\text { Found in } \% \\
\text { to substance } \\
\text { concentration } \\
\text { of the } \\
\text { comparison }-Y_{i}, \%\end{array}$ & $\begin{array}{l}\text { Found in } \% \\
\text { to presented } \\
\mathrm{Z}_{\mathrm{i}}=100^{*}\left(\mathrm{Y}_{\mathrm{i}} / \mathrm{X}_{\mathrm{i}}\right), \%\end{array}$ \\
\hline 1 & 0.0307 & 61.42 & 0.182 & 61.27 & 99.76 \\
\hline 2 & 0.0357 & 71.54 & 0.246 & 71.16 & 99.46 \\
\hline 3 & 0.0396 & 79.21 & 0.301 & 78.49 & 99.09 \\
\hline 5 & 0.0475 & 95.01 & 0.434 & 94.35 & 99.30 \\
\hline 6 & 0.0509 & 101.70 & 0.505 & 102.46 & 100.74 \\
\hline 7 & 0.0564 & 112.98 & 0.624 & 114.25 & 101.12 \\
\hline 8 & 0.0608 & 121.56 & 0.723 & 122.80 & 101.02 \\
\hline 9 & 0.0669 & 133.76 & 0.867 & 133.80 & 100.03 \\
\hline \multicolumn{3}{|l|}{ Mean, $\bar{Z}, \%$} & 99.93 & & \\
\hline \multicolumn{3}{|c|}{ Relative standard deviation, sz\% } & 0.84 & & \\
\hline \multicolumn{3}{|c|}{ Critical value for repeatability $\Delta \% \leq$} & 2.5 & & \\
\hline \multicolumn{3}{|c|}{$|\bar{Z}-100|$} & 0.07 & & \\
\hline \multicolumn{6}{|c|}{ Systematic error $\delta=\left.\right|^{2-100 \mid}$} \\
\hline \multicolumn{3}{|c|}{ Criterion of insignificance of systematic error $\delta \leq \Delta / 3=2.5 / 3=0.83$} & in progress & & \\
\hline \multicolumn{3}{|c|}{ General conclusion to the method: } & Correct & & \\
\hline
\end{tabular}

Table 4: Results of in-laboratory precision testing

\begin{tabular}{|c|c|c|c|}
\hline \multirow[t]{2}{*}{ Solution No. } & \multicolumn{3}{|c|}{ Values $\mathrm{Z}_{\mathrm{i}}$} \\
\hline & 1 study & 1 study & 1 study \\
\hline 1 & 99.82 & 99.21 & 99.88 \\
\hline 2 & 99.45 & 98.28 & 99.07 \\
\hline 3 & 98.21 & 99.16 & 98.72 \\
\hline 4 & 99.33 & 98.37 & 99.01 \\
\hline 5 & 98.16 & 99.14 & 98.25 \\
\hline Mean & 98.99 & 98.83 & 98.99 \\
\hline Combined mean value $\mathrm{Z}_{\text {intr }} \%$ & 98.94 & & \\
\hline $\mathrm{S}_{\text {z }}(\%)$ & 0.76 & 0.46 & 0.59 \\
\hline $\mathrm{SD}_{\mathrm{z}}(\%)$ & 0.16 & & \\
\hline$\Delta^{2} \%(\mathrm{k}=5)$ & \multicolumn{3}{|c|}{$=1.76 * 0.16=0.28 \leq 1.75$} \\
\hline
\end{tabular}

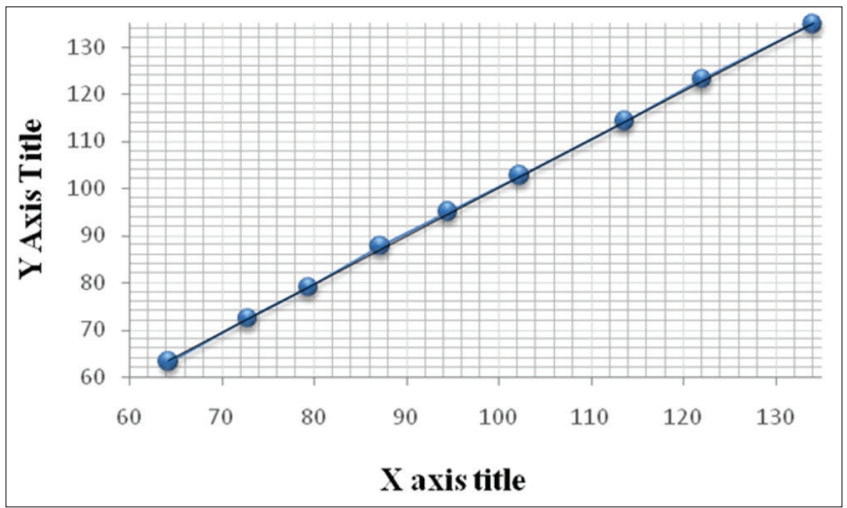

Fig. 1: Linear dependence of optical density on the concentration of (S)-2,6-diaminohexanoic acid of 3-methyl-1,2,4-triazolyl-5thioacetate in normalized coordinates

magnitude of optical density. The influence of these factors was taken into account when choosing the best conditions for determination.

As a result of the work, it was proved that the method of quantifying the determination of the (S) -2,6-diaminohexanoic acid of 3-methyl-1,2,4triazolyl-5-thioacetate according to characteristics such as specificity, linearity, correctness, range of use, precision, and robustness are valid.
The developed method of quantitative determination of substance by absorption spectrophotometry method in the ultraviolet region will be further used to develop methods for the analysis of tablet formulations of (S) -2,6-diaminohexanoic acid of 3-methyl-1,2,4-triazolyl-5thioacetate.

\section{CONCLUSION}

In the course of the work analyzed the data of scientific literature on the prevalence of the incidence of the Ukrainian population of CHD, the prospects for the creation of new metabolito tropic cardioprotective drugs. The procedure of validation of the method of quantitative determination of angiolin in substance by spectrophotometric method is carried out. A forecast of the maximum permissible uncertainty of the analysis is conducted, the results of which satisfy the requirements of the (SPF) State Pharmacopoeia of Ukraine. Validation characteristics are investigated for the method: stability, robustness, linearity, correctness, convergence, and reproducibility. The results prove that the technique can be properly restored and suitable for future use.

\section{AUTHOR'S CONTRIBUTION}

The article is a product of the intellectual environment of the whole team and that all members have contributed in various degrees to the analytical methods used, to the research concept, and to the experiment design.

\section{CONFLICTS OF INTEREST}

All authors have none to declare.

\section{REFERENCES}

1. Sundström J, Arima H, Woodward M, Neal B. Blood pressure-lowering treatment based on cardiovascular risk: A meta analysis of individual patient data. Lancet 2014;384:591-8.

2. White W. Blood Pressure Monitoring in Cardiovascular Medicine and Therapeutics. New Jersey: Humana Press; 2001.

3. Mazur I, Chekman I, Belenichev I. Metabolithotropic Drugs. Ukraine: Zaporozhye; 2007

4. Kucherenko LI, Khromylova OV, Moriak ZB, Tkachenko GI, Vaschenko OV. Stage control of tablets manufacturing. Aktualnipytanniafarmatsevtychnoi i Medychnoinaukytapraktyky 2014;2:31-4

5. Mazur IA, Belenichev IF, Kucherenko LI, Georgievskij GV, Chekman IS, Buchtiyarova TA, et al. Approaches to the Development 
and Creation of Metabolitotropnyh Drugs-Derivatives of 1,2,4-Triazole. Gurzuf: Proceedings of the $12^{\text {th }}$ Scientific and Practical Seminar; 2012.

6. Ukrainian Scientific Pharmacopoeial Center for Quality of Medicines. State Pharmacopoeia of Ukraine. $2^{\text {nd }}$ ed., Vol. 1. Kharkiv: Ukrainian Scientific Pharmacopoeial Center for Quality of Medicines, Ukrainian; 2015. p. 85-100.

7. Beckett AH, Stenlake JB. UV-Visible Spectrophotometry: Practical Pharmaceutical Chemistry. $4^{\text {th }}$ ed., vol. 2. New Delhi: C.B.S. Publishers; 2001. p. 285-97.
8. Kucherenko L. On the standardization of hypertril substance. Pharm Rev 2015;2:64-7.

9. Manjunath S, Chouhan V, Sandeep S. Spectrophotometric estimation of levosulpiride in bulk drug and formulations. Int J Pharm Pharm Sci 2011;3:135-7.

10. Nayak SC, Kulkarni PV, Bhaskar V, Chavhan V. Development and validation of UV spectrophotometric method for simultaneous estimation of aceclofenac and pantoprazole in bulk and tablet dosage forms using hydrotropic solvent. Int J Pharm Pharm Sci 2013;5:390-3. 